\title{
¿Qué opina el profesorado cuando recibe formación en el Modelo de Responsabilidad Personal y Social? Un estudio basado en grupo de discusión What is professors' opinion when they receive training on the Personal and Social Responsibility Model? A study based on focus group
}

Ángel Ibaibarriaga Toset, Carlos M ${ }^{\mathrm{a}}$ Tejero-González

Universidad Autónoma de Madrid (España)

\begin{abstract}
Resumen. Los programas basados en el Modelo de Responsabilidad Personal y Social (TPSR) fueron diseñados para que adolescentes y jóvenes de contextos desfavorecidos tomen consciencia de su propio desarrollo personal y social, utilizando para ello la práctica estructurada de actividades físico-deportivas. En el presente estudio, seis docentes de educación física seleccionados mediante muestreo incidental y criterios de inclusión recibieron un plan formativo en TPSR. El estudio siguió un diseño cualitativo de grupo de discusión. Los testimonios que emergieron fueron analizados a partir de cuatro categorías: características estructurales del plan de formación, contenidos teórico-prácticos, perfil del formador y valoración global del plan formativo. Los resultados sugieren que el programa formativo debe contar al menos con 50 horas y que es necesaria la adecuación de la formación a las características contextuales de los docentes y sus centros educativos en relación con la composición del grupo, la elección de horarios y las localizaciones. Asimismo, se destaca que el formador debe conocer en profundidad el TPSR y hacer uso de un estilo de enseñanza motivador y entusiasta, a través de una metodología activa caracterizada por un enfoque práctico. Por último, los participantes concluyeron sobre la importancia de una formación continua que pueda extenderse a las diferentes áreas del currículo y etapas educativas.
\end{abstract}

Palabras clave: Modelo de responsabilidad personal y social, educación física, formación profesorado.

\begin{abstract}
Programs based on the Personal and Social Responsibility Model (TPSR) were designed to target adolescents and young people from disadvantaged backgrounds with the objective of making them aware of their own personal and social development through the practice of physical activities and sports. In the present study, six Physical Education teachers selected using incidental sampling and inclusion criteria were trained in a TPSR training plan. The study, following a qualitative approach based on focus group, analysed the data according to the following categories: Structural characteristics of the training plan, theoretical and practical content, teacher profile, and overall evaluation of the educational programme. The results of the study suggest that the training process should take at least 50 hours, an individualized education plan being strictly necessary, with aspects such as context, school centre, groups selection, timetables, and location needing to be considered. In the same way, a deep knowledge of the TPSR model alongside with a motivating and active methodology based on practical approach was emphasized. Finally, the participants highlighted the importance of continuous training so to transfer the model to other subjects within the Primary Education curriculum.
\end{abstract}

Key words: Personal and Social Responsibility Model, physical education, Educational personnel training.

\section{Introducción}

En este estudio se aborda el denominado Modelo de Responsabilidad Personal y Social, también conocido por su acrónimo TPSR derivado de lengua inglesa (Teaching Personal and Social Responsibility). El modelo TPSR fue diseñado por Don Hellison (1995, 2003, 2011), siendo un programa que se fundamenta en la idea de que los jóvenes, por medio de la práctica de actividad físico-deportiva estructurada, asuman la responsabilidad personal de su propio desarrollo y optimicen un comportamiento social deseable. Con dicha finalidad educativa, las primeras aplicaciones del TPSR se realizaron en los años 70 del siglo XX en barrios marginales de la ciudad de Chicago (EE.UU.), concretamente, en escuelas alternativas, en centros de menores y en actividades extraescolares con adolescentes y jóvenes en riesgo de exclusión, caracterizados por conductas violentas, problemas de drogadicción y alcoholismo. En definitiva, el objetivo del programa TPSR era dotar a estos jóvenes de experiencias que favorecieran la oportunidad de desarrollar sus capacidades personales, sociales y su responsabilidad, con el fin de erradicar conductas no saludables desde un punto de vista físico, psicológico y social. Así, el modelo

Fecha recepción: 02-11-18. Fecha de aceptación: 18-07-19

Ángel Ibaibarriaga Toset

angel.ibaibarriaga@uam.es
TPSR busca la adquisición de unos valores que promuevan la decencia humana, potenciando el desarrollo holístico y el establecimiento de relaciones positivas entre las personas (Hellison, 2011). Y al mismo tiempo dotando a niños y jóvenes de experiencias de éxito que les ofrezcan la oportunidad de desarrollar sus capacidades personales y sociales, aprendiendo a ser responsables de sí mismos y de los demás e incorporando las estrategias que les permitan ejercer el control de sus vidas.

El modelo se estructura en niveles por los que el alumnado participante debe transitar, siendo un modelo gradual y flexible que permite la reminiscencia de algún nivel superado, pudiendo retornar a niveles anteriores si el aplicador lo cree necesario. El nivel 0 o de irresponsabilidad se caracteriza por la presencia en el alumnado de comportamientos carentes de responsabilidad, justificando sus conductas en las acciones de otros. El nivel 1 o de respeto por los derechos y los sentimientos de los demás tiene la finalidad de crear en el aula un clima de confianza y seguridad a nivel físico y psicológico, haciendo uso de la negociación y el diálogo para resolver discrepancias, desavenencias y conflictos y siempre mostrando respeto a las cualidades y características de todos los compañeros. El nivel 2 o de participación y esfuerzo tiene el objetivo de promover la participación activa y esforzada en las actividades, fomentando experiencias positivas donde se otorgue valor a la buena disposición ante el trabajo y mostrando interés hacia las tareas propuestas independientemente del nivel de afinidad hacia las mis- 
mas. El nivel 3 o de autonomía personal trata de incentivar al alumnado para que aprenda a ser independiente y asumir responsabilidades con capacidad de autogestión y planificación del propio aprendizaje sin la acción directa del profesorado. El nivel 4 o de ayuda a los demás y liderazgo insiste en la empatía y el compromiso moral de ayuda a otros sin esperar recompensa externa. Y el nivel 5 o de transferencia persigue que los niveles anteriores sean aplicados a otros ámbitos de la vida personal fuera del aula como, por ejemplo, familia y amigos, nuevos entornos, etc.

A partir del TPSR han surgido programas directamente relacionados con éste que se han aplicado en diferentes países, tipos de población y en distintos contextos. A nivel internacional, podemos destacar investigaciones realizadas desde el área de educación física: Compagnone (1995), Debusk \& Hellison (1989), Gülay \& Gürsel (2017), Kallusky (2000), Pavão, Santos, Wright \& Gonçalves (2019) y Wright, Li, Ding \& Pickering (2010). Y también se observan un gran número de investigaciones llevadas a cabo en el marco de la actividad física y el deporte extraescolar: Gordon, Jacobs \& Wright (2016), Kahne, Nagaoka, Brown, O’Brien, Quinn \& Thiede (2001), Lee \& Martinek (2009), Martinek \& Hellison (2009), Martinek, McLaughlin \& Schilling (1999), Schilling (2001), Walsh (2007,2008), Walsh, Ozaeta \& Wright (2010) y Wright, White \& Gaebler-Spira (2004).

En el contexto del Estado Español, como ocurre a nivel internacional, existen investigaciones dentro de la asignatura de educación física: Escartí, Gutiérrez, Pascual \& LlopisGoig (2010), Escartí, Pascual \& Gutiérrez (2005), Gómez-Mármol (2014), Marín (2011), Martínez (2011), Sánchez-Alcaraz, Gómez-Mármol, Valero, De la Cruz \& Esteban (2012) y Sánchez-Alcaraz, Gómez-Mármol, Valero \& Dela Cruz (2013). Y otros estudios de carácter más global: Belando \& FerrizMorell (2012), Cecchini, Montero \& Pena (2003), Escartí et al. (2006), Jiménez (2000), Jiménez y Durán (2004), Pardo (2008), Ruiz et al. (2006) y Vizcarra (2004).

Más allá del contexto, Estado Español u otros países, educación física escolar o una actividad físico-deportiva más general, la mayoría de las anteriores investigaciones estaban orientadas a conocer los efectos alcanzados por el Modelo de Responsabilidad sobre los participantes en distintas variables como el respeto, el autocontrol, la implicación, el esfuerzo, la autonomía, la cooperación, la autoeficacia, la integración social, la responsabilidad personal y social y la motivación. Si bien, en los últimos años han proliferado los estudios cuya finalidad también ha sido conocer diferentes aspectos de la aplicación del TPSR que repercuten directamente en los resultados, como la fidelidad de implementación del modelo por parte de los aplicadores (Escartí, Gutiérrez, Pascual \& Wright, 2013; Escartí, Llopis-Goig \& Wright, 2018; Pascual et al., 2011), la percepción del modelo por parte de los implementadores (Llopis-Goig, Escartí, Pascual, Gutiérrez \& Marín, 2011; Sánchez-Alcaraz, Cañadas, Valero, GómezMármol \& Funes, 2019; Walls, Veri \& Willard, 2015), el análisis de las estrategias empleadas por los docentes (Beaudion, 2012) el impacto de la aplicación del modelo en la práctica docente del profesorado implementador (Lee \& Choi, 2015) e incluso la asociación del Modelo de Responsabilidad con otros modelos pedagógicos como el Modelo de Iniciación Deportiva (Fernández-Río \& Menéndez-Santurio, 2017;
Menéndez \& Fernández-Río, 2016) y el Aprendizaje Cooperativo (Fernández-Río, 2014).

$\mathrm{Al}$ respecto, cabe destacar que algunas de las anteriores investigaciones han incidido sobre la importancia de la formación del profesorado implementador en aspectos relacionados con la naturaleza del TPSR (Llopis-Goig et al., 2011) y la necesidad de llevar a cabo nuevos estudios sobre los programas de entrenamiento (Pascual et al., 2011), analizando los elementos clave que deben ser parte de dicha formación (Escartí et al., 2012). En virtud de lo anterior, el objetivo del presente estudio fue conocer la valoración de un programa de formación orientado a la aplicación del TPSR, a través de las percepciones del profesorado participante.

\section{Método}

\section{Diseño y aspectos éticos}

La metodología empleada ha sido de carácter cualitativo, enfocada a la comprensión de fenómenos educativos para elaborar procesos de mejora en la enseñanza y el aprendizaje (Svensson \& Doumas, 2013). La investigación se enmarca dentro de un paradigma interpretativo, fundamentado en la búsqueda del conocimiento de un objeto de estudio en su contexto, sin la pretensión de obtener resultados generalizables y estandarizados. El estudio fue aprobado por el comité de ética de la investigación de la universidad a la que pertenecen los autores de este trabajo.

\section{Participantes}

Participaron en el estudio un total de seis docentes, todos ellos funcionarios de carrera del cuerpo de maestros en la especialidad de educación física, que desarrollaban su profesión en la Comunidad de Madrid. El grupo estuvo formado por dos mujeres y cuatro hombres con una media de edad $38 \pm 5.97$ años, con edades comprendidas entre los $32 \mathrm{y}$ los 50 años y una experiencia profesional entre los nueve y 28 años. La selección de los participantes se realizó a través de un muestreo incidental acorde a una serie de criterios de inclusión (Heinemann, 2003): (1) impartir docencia directa en la etapa de educación primaria en un centro de titularidad pública, (2) continuidad durante los dos cursos siguientes en el mismo centro educativo, (3) participar en un curso de formación sobre aspectos relacionados con el TPSR y (4) disponer de autorización por parte del consejo escolar y la dirección del centro educativo a llevar a cabo la aplicación del TPSR. Así mismo, en cuanto al procedimiento de selección, se llevó a cabo una reunión informativa en las instalaciones municipales del Ayuntamiento de Parla (Madrid), donde asistieron maestros especialistas en educación física procedentes de diferentes centros educativos públicos de la zona. Se les informó del proceso de investigación, sus objetivos y de la confidencialidad y anonimato de los resultados, invitándoles a participar en la investigación. Por último, cuando los profesores confirmaron su participación, se proporcionó esta información a los equipos directivos de sus respectivos centros y se obtuvo la autorización pertinente a través de la firma de un consentimiento informado.

\section{Procedimiento}

Todos los participantes recibieron un plan de formación 
sobre el marco conceptual del TPSR y las estrategias para su desarrollo, estructurado en cinco bloques de contenido: (1) niveles de responsabilidad, (2) modelo de sesión de trabajo, (3) perfil del docente aplicador, (4) estrategias de enseñanza de responsabilidad y resolución de conflictos y (5) entrenamiento de habilidades sociales y de comunicación. Esta formación tuvo un carácter teórico-práctico, con una duración de 50 horas que se extendió a lo largo de un curso escolar entre los meses de octubre y mayo. La periodicidad fue de una sesión semanal de 75 minutos de duración. El lugar de celebración de las sesiones fue el Colegio de Educación Infantil y Primaria La Paloma, situado en la localidad de Parla (Madrid). La elección del lugar tuvo en consideración la cercanía a los centros de trabajo del profesorado participante, facilitando de este modo el acceso a la formación. La persona encargada de impartir la formación fue el investigador principal del estudio. Por último, destacar que el plan formativo quedó integrado dentro del Plan de Formación Permanente del Profesorado de la Comunidad de Madrid, a través del Centro Territorial de Innovación y Formación (CTIF) Madrid-Sur, en la modalidad de grupos de trabajo, con el reconocimiento de 2 créditos de formación.

Posteriormente a la formación, se llevó a cabo un grupo de discusión semi-estructurado con preguntas abiertas, el cual se desarrolló siguiendo los procedimientos sugeridos por expertos en relación al número de participantes, la duración de las sesiones, el lugar de reunión, el inicio y desarrollo de la misma, la formulación y estructuración de las preguntas y la grabación de la información (Callejo, 2001; Gutiérrez, 2008; Ibáñez, 2003; Kitzinguer \& Barbour, 1999; Krueger, 1991). Concretamente, dicho grupo de discusión se llevó a cabo en el mes de junio a la finalización del desarrollo del plan formativo. El lugar de celebración fue el mismo en cual se impartieron las sesiones de formación, adaptándose de este modo al perfil de los participantes (Canales \& Peinado, 1994). Se optó por la elección de un moderador externo a los investigadores principales, con el objeto de no influir en las respuestas del profesorado y conseguir explicaciones más detalladas (Barbour, 2007), dado que el equipo de investigación había sido el encargado de impartir la formación. El grupo de discusión fue grabado en audio y en vídeo, con una duración de 63 minutos. El moderador planteaba una pregunta y los docentes iban participando aleatoriamente, reconduciendo el contenido argumental cuando las valoraciones se alejaban del plano del estudio.

\section{Análisis de la información}

Con el objeto de llevar a cabo el análisis de contenido del grupo de discusión, se elaboró previamente un sistema de categorías provisional (Barbour, 2007) basándose en una revisión bibliográfica exhaustiva y en los bloques temáticos del guion establecido para la puesta en acción del grupo de discusión. Posteriormente se realizó una lectura minuciosa de la transcripción con el fin de obtener una comprensión global de su contenido (Friberg \& Öhlen, 2007), seguido de una fragmentación de los datos en unidades mínimas de significado. El sistema de categorías definitivo se alcanzó mediante consenso y triangulación de tres analistas (Vallejo \& Mineira, 2009), quienes procedieron con razonamiento deductivo, generando categorías y subcategorías soporta- das teóricamente e inductivo, con categorías y subcategorías emergentes en base al contenido del grupo de discusión (Coffey \& Atkinson, 2005). Finalmente, el sistema quedó estructurado en cuatro categorías principales: (1) características estructurales del plan de formación, (2) contenidos teóricos-prácticos, (3) perfil del formador y (4) valoración global del plan formativo. En la Tabla 1 se muestran estas categorías con las subcategorías correspondientes. Asimismo, se recurrió a la estrategia de revisión por parte de los participantes member check, por medio de la cual los participantes pudieron comprobar la fidelidad de los textos transcritos (Patton, 2002). La codificación y la saturación de los datos se llevó a cabo mediante análisis de patrones cruzados (Saldaña, 2009)

\begin{tabular}{|c|c|c|c|}
\hline Código & Categoría & Código & Subcategoría \\
\hline \multirow{6}{*}{$\mathrm{CE}$} & \multirow{6}{*}{$\begin{array}{l}\text { Características } \\
\text { Estructurales del } \\
\text { programa de } \\
\text { formación }\end{array}$} & CEdc & Duración total curso formación \\
\hline & & CEfs & Frecuencia sesiones \\
\hline & & CEds & Duración cada sesión \\
\hline & & CEme & Materiales empleados \\
\hline & & CEiu & Instalaciones utilizadas \\
\hline & & CEta & Tipología de agrupamientos \\
\hline \multirow{6}{*}{ СT } & \multirow{6}{*}{$\begin{array}{l}\text { Contenidos Teórico- } \\
\text { prácticos }\end{array}$} & CTacc & Adaptación al contexto del centro \\
\hline & & CTina & Información necesaria para la aplicación \\
\hline & & CTocc & Opinión sobre contenido del curso \\
\hline & & Ctaf & Aspectos de contenido que faltan \\
\hline & & СТср & Conocimientos previos \\
\hline & & CTfc & Necesidad de Formación Continua \\
\hline \multirow{5}{*}{ PF } & \multirow{5}{*}{ Perfil del Formador } & PFme & Metodología empleada \\
\hline & & PFps & Preparación sesiones \\
\hline & & Pfcm & Conocimiento materia \\
\hline & & PFap & Atención a participantes \\
\hline & & PFcc & Conocimiento del contexto \\
\hline \multirow{4}{*}{ VG } & \multirow{4}{*}{ Valoración Global } & VGap & Aspectos destacar positivamente \\
\hline & & VGpm & Propuestas mejora \\
\hline & & VGb & Valoración global \\
\hline & & VGep & Expectativas personales \\
\hline
\end{tabular}

\section{Resultados}

Del grupo de discusión emergieron 98 extractos de contenido, los cuales fueron estructurados de acuerdo a las cuatro categorías establecidas previamente en la Tabla 1: (1) características estructurales del plan de formación, (2) contenidos teórico-prácticos, (3) perfil del formador y (4) valoración global del plan formativo. A continuación se muestran por categorías los extractos más significativos y coincidentes.

Características estructurales del plan de formación

El 26,53\% de los extractos $(n=26)$ hizo referencia al plan de formación. En lo referente a la duración de la formación (50 horas), aunque no existe una opinión unánime, la mayor parte del profesorado participante indica que les parece adecuada:

La duración a mí me ha parecido buena, hemos tenido tiempo suficiente a lo largo del curso, se trata de conocer la teoría que soporta el programa (TPSR) y en eso hemos tenido suficiente, por mi parte muy bien.

Sin embargo, uno de los docentes indica la necesidad de realizar una formación de mayor duración:

Yo voy a decir que me parece corto, porque a nivel teórico sí, pero es cierto que a nivel práctico se quedan algunas cosillas que pueden surgir alguna duda [...] Lo hubiera alargado algo más en el tiempo el curso, empezando antes y acabando después.

Ante esta sugerencia el resto de componentes del profe- 
sorado muestra su rechazo a través de diferentes argumentos, destacando de manera unánime problemas de calendario:

Prolongarlo en el tiempo yo no lo veo, luego te metes en mayo con otras actividades propias de la Educación Física, que si excursiones, olimpiadas... viene el trabajo más concentrado con el buen tiempo y me parece bien terminarlo en mayo y no meternos en junio.

Donde sí aparece un consenso es en la frecuencia semanal de las sesiones, destacan que este formato permitía tener de manera permanente frescura en los contenidos y daba lugar a poner en común experiencias que surgían en el desarrollo de actividades y tareas que llevaban a cabo en sus respectivos centros:

Intensificar a dos sesiones semanales sería prácticamente inviable, con lo cual una sesión semanal está bien, porque si lo alargas a una frecuencia quincenal, de una sesión a otra no te acuerdas, por eso una sesión semanal me parece la mejor frecuencia [...]

Es necesario juntarse una vez por semana, tratar las cosas que van surgiendo, sobre todo esos temas que son más prácticos

En cuanto a la duración de las sesiones (1h 15’), destacan como aspecto muy positivo que se hayan llevado a cabo dentro del horario lectivo, en concreto en la hora de dedicación exclusiva que tiene el profesorado en los centros, facilitando de este modo la asistencia a las sesiones:

Yo creo que el tiempo de la exclusiva era el adecuado, ese tiempo nos ha permitido aclarar bien los conceptos, se han solventado las dudas que surgían y tampoco hemos ido con excesivo agobio, por mi parte bien [...]

Darle más tiempo es imposible porque no lo hay, implica ya tiempo personal, por la tarde todos tenemos muchas cosas que dejan poco tiempo libre.

Un aspecto valorado muy positivamente ha sido la composición del grupo de docentes participantes, exponiendo de forma explícita que ser un grupo reducido ha facilitado en gran manera la participación a lo largo de toda la formación:

Lo que también ha favorecido, es que al ser compañeros de pocos colegios ha dado lugar a participar más, hemos cogido pronto confianza entre todos, que si hubiéramos sido 20 personas no hubiera dado lugar, ser un grupo más o menos reducido ha favorecido la participación.

En esta misma línea, argumentan que proceder de centros escolares con un contexto socio-económico de similares características ha sido un factor importante en el desarrollo de la cohesión del grupo, favoreciendo la participación durante el proceso de formación:

Si hubiera habido más colegios te enriqueces de más experiencias, pero te da lugar a participar menos. Lo que es muy positivo, es que al estar casi todos muy cerca, nos nutrimos de una población muy parecida [...]

Eso es algo muy positivo que hemos tenido, que los centros tienen características muy parecidas. Los mismos problemas que puede tener Ángel (Investigador principal) aquí son los que puedo tener yo.

Contenidos teórico-prácticos

El 37,5\% de los extractos $(n=36)$ estuvieron vinculados a los contenidos teórico-prácticos. El primer aspecto destacable es que ninguno de los docentes participantes tenía con anterioridad ningún tipo de formación en el ámbito del TPSR. Alguno de ellos tenía constancia de la existencia del Modelo de Responsabilidad, pero sin conocer sus características y modo de aplicación:

Me llamó la atención cuando Ángel comentó que se estaba poniendo en práctica desde hace más de treinta años, pero yo aquí no había oído nada. [...]

En mi caso había leído algo sobre el tema, pero no lo conocía en profundidad.

En lo referente a la aplicabilidad de los contenidos al contexto particular de cada uno de los centros de procedencia, la opinión es unánime. Todos los participantes aseguraron que se ajustan a las necesidades que tienen y que les otorga posibles soluciones a diversas problemáticas que presenta sus aulas de referencia:

Responde un poco a las dificultades que nos podemos encontrar en nuestro centro, al tener tanta diversidad cultural, tantas nacionalidades, tantos niños de diferentes procedencias, tanta problemática socio-familiar, sí que se adecúa $[. .$.

Creo que una de las mejores cosas que tiene este curso que hemos hecho, es que tiene una aplicación directa en nuestros cursos y nuestras clases. Aquí surgen muchos conflictos por esa diversidad que se comenta y tener una herramienta para resolver esos problemas y conflictos es bastante bueno.

Por otra parte, exponen de forma mayoritaria que el nivel de contenidos teórico-prácticos adquiridos les hacen sentir lo suficientemente formados para iniciar la aplicación del TPSR. Sin embargo, mostraron dudas sobre estar preparados para poder dar respuesta a las posibles contingencias que puedan surgir durante la implementación del modelo:

Para empezar, me veo preparado, es cierto que la práctica seguro que nos van a surgir muchas más cosas y vamos a necesitar más material y más ayuda, pero yo creo que ahora mismo sí, aunque luego pueda surgir alguna dificultad más [...]

En la parte teórica no hecho nada en falta, es más en lo práctico que pueda surgir alguna duda más adelante. Poner el modelo en las clases e iniciar el programa lo veo factible, luego hay que ver si surgen cosillas y podemos dar soluciones.

En relación con el aspecto anterior, los docentes argumentan la necesidad de realizar una formación continua durante el proceso de aplicación del TPSR que les permita poner en común dificultades encontradas y otorgar posibles soluciones:

Creemos que es necesario continuar con una formación continua, esta base teórica está bien, pero sí que nos vemos un poco indefensos a la hora de plantearnos en qué nivel estamos, si estoy en el uno o estoy en el dos, o algunos en el uno y otros en el dos, yo creo que la práctica es la que realmente nos puede decir si lo estamos haciendo bien o no [...]

A nivel de teoría me veo bien, pero a nivel práctico a lo mejor estoy un poco pez, aplicar estrategias como el banco de la paz u otras sí, pero aplicar el programa entero desde el principio no. Por eso veo muy bueno y necesario que el año que viene podamos seguir con una formación continua. 
Perfil del formador

El 20,83\% de los extractos ( $n=20$ ) hicieron referencia al perfil del formador. La primera variable que destacan los docentes-participantes de manera unánime fue la importancia que tuvo el conocimiento por parte del formador de la realidad de sus centros, exponiendo que este hecho supuso que gran parte de los contenidos teórico-prácticos trabajados se hayan contextualizado en situaciones que reflejan su realidad educativa:

Siempre los contenidos teóricos venían acompañados de ejemplos prácticos del día a día del aula. A lo mejor no práctico de que nosotros tuviéramos que hacer algo, pero casi todo lo que hemos visto venía acompañado de ejemplos reales que nos pasan a todos [...]

Que conozca los colegios en los que trabajamos suponía que en el tema de los ejemplos nos viéramos reflejados y el programa nos invitaba a pensar «es que esto lo estamos sufriendo todos los días»

En lo referente al nivel de conocimientos en el ámbito del TPSR que posee el formador, no otorgan tanto valor el dominio de la materia, sino que destacan lo importante que ha sido el sentimiento de credibilidad en el modelo, en sus potencialidades y en las posibilidades de éxito que ha transmitido a la hora de enseñarlo durante toda la formación:

Te hace partícipe de lo que estás viendo, como él está convencido y cree en lo que te está explicando, te ayuda también a motivarte [...]

Transmite muy bien los conocimientos, es lo que han apuntado los compañeros, conoce el programa, cree que el programa da resultados y nos lo ha transmitido [...]

A mí positivamente me ha gustado como nos ha transmitido todo, se nota que controla y lo ha transmitido de una manera que se nota que cree en ello, que le gusta su trabajo y eso contagia.

Por último, en cuanto a la metodología empleada, les parece positiva la combinación de aprendizajes teóricos con actividades de carácter práctico, sin embargo, una parte importante de los docentes demanda la necesidad de aumentar la carga práctica a lo largo del proceso de formación:

Ha sido una combinación de metodología teórico-práctica, por lo cual en ningún momento se hacía muy pesado, además eran muy participativas las sesiones [...]

En cuanto a práctico, también hubo partes en la que tuvimos que poner aspectos en práctica, como cuando tuvimos que grabarnos, a mí me encantó, luego pusimos muchas cosas en común. Me hubiera gustado más sesiones de ese tipo, porque si es verdad que ha habido más carga teórica, pero siempre muy ameno.

Valoración global del plan formativo

El 16,66\% de los extractos $(n=16)$ refirieron a valoraciones globales del plan formativo, siendo una valoración muy positiva y satisfechas con todo el proceso formativo, exponiendo en varios casos que han generado nuevas expectativas de cara a la aplicación del TPSR:

Para mí ha sido muy positivo realizar este curso, una vez que empezamos a ver las herramientas, los niveles... ya dije ; ah! Si esto es lo que yo busco, lo que pasa es que me he hecho más expectativas para el año que viene (todos asienten), este año pones en acción estrategias, pero el año que viene pones en marcha todo el programa y lo vas visualizando un poquito y ves soluciones a problemas que tienes.

Un aspecto importante que han reseñado todos los docentes como propuesta de mejora, es la posibilidad de extender la formación a otras áreas del currículo de la etapa de Educación Primaria, de manera que no quede circunscrita únicamente al área de Educación Física:

Yo destacaría como negativo, el que solo lo enfoquemos a la Educación Física, a lo mejor lo ha enfocado solo a la Educación Física porque es el ámbito que nos concierne a nosotros, pero lo que es cierto, que como no solo impartimos Educación Física casi todos, es muy aplicable a otras áreas, hacerlo extensible a nivel de centro y que se pudiera llevar a cabo por todos los profesores [...]

Una propuesta de mejora para un segundo año sería realizar un protocolo para hacer una transferencia a otras áreas del centro.

\section{Discusión}

De acuerdo con el modelo de responsabilidad y sus características, la figura del profesor es un elemento clave para provocar en el alumnado los aprendizajes necesarios para su desarrollo personal y social (Escartí et al., 2010; Pascual et al., 2011). Por ejemplo, algunos de los factores que en gran medida van a determinar el éxito del modelo son el clima de aula establecido, la puesta en acción de las estrategias de responsabilidad, la capacidad de otorgar liderazgo y la posibilidad de crear situaciones de éxito para los estudiantes. En este sentido, la gestión coherente por parte del profesorado de los distintos elementos vertebradores del TPSR está directamente relacionada con la formación recibida. Así, recientemente se ha llegado a la conclusión de que la posibilidad de producir resultados positivos en el aprendizaje personal y social se debe a la aplicación del modelo con eficacia, para lo cual es imprescindible un formación estructurada y contextualizada a las condiciones particulares del profesorado y de los centros educativos de aplicación (Pozo, GraoCruces \& Pérez-Ordas, 2016).

En esta investigación se ha conocido la valoración del profesorado participante en un plan de formación de aspectos relacionados con el TPSR. A nivel logístico y estructural, los docentes han expuesto que una formación efectiva debe tener una carga lectiva aproximada de 50 horas, cumpliendo en la medida de los posible una serie de características que se ajustan a sus condiciones laborales: (1) frecuencia de una sesión a la semana, (2) duración no superior a los 90 minutos, (3) desarrollo dentro de la jornada lectiva, preferentemente en la franja horaria de obligada permanencia en el centro tras la docencia directa, (4) grupos reducidos que favorezcan la participación y (5) recibir la formación en instalaciones cercanas a los centros educativos de referencia, facilitando así la asistencia. Es evidente que estas preferencias expuestas por parte de los docentes están muy condicionadas por sus escenarios laborales y las particularidades propias de los centros educativos de titularidad pública, razón por la que varias de estas condiciones difieren de investigaciones precedentes, donde en muchos casos el periodo de formación era menos extenso (20-30 horas) y se concentraba en menos sesiones de mayor duración (no inferiores a 2 horas). Por lo 
tanto, los aspectos estructurales de un proceso de formación en el modelo de responsabilidad van a estar muy condicionados por las características que presenten los profesores aplicadores.

En cuanto a la adecuación de los contenidos teóricoprácticos: niveles de responsabilidad, modelo de sesión de trabajo, perfil del docente aplicador, estrategias de enseñanza de responsabilidad y resolución de conflictos y entrenamiento de habilidades sociales y de comunicación, se ha constatado que se ajustan adecuadamente al contexto de aplicación (Llopis-Goig et al., 2011). Además, los docentes expusieron su preferencia hacia una formación basada en actividades y experiencias prácticas, utilizando estrategias más participativas (Escartí, et al., 2013), y de menor carga teórica, demandando que se mantenga la formación durante el periodo de implementación del TPSR, algo frecuente en la aplicación del Modelo de Responsabilidad. Esta preferencia hacia una formación eminentemente práctica, requiere de modificaciones en el modelo de formación. Estudios precedentes (Sánchez-Alcaraz et al., 2013; Escartí et al., 2010) optan por un formato intensivo, en el cual se desarrollan todos los aspectos propios del modelo en pocas sesiones de larga duración. En este estudio, se ha preferido desarrollar una formación de mayor duración en el tiempo, temporalizando las sesiones de forma semanal, lo que ha permitido incorporar aprendizajes de carácter práctico, desarrollados en situaciones reales con características similares a los futuros contextos de aplicación del modelo, generando procesos de reflexión y debate entre los docentes participantes y enriqueciendo de esta manera el proceso formativo por medio de un feedback permanente. Por otro lado, queda patente la necesidad de establecer una formación continua durante el periodo de aplicación del TPSR, mediante la cual, se facilite asesoramiento al profesorado y retroalimentación sobre el desarrollo del modelo, además de un espacio de reunión. Así mismo, los docentes han realizado alusiones a la posibilidad de orientar la formación a la aplicación del TPSR en otras áreas curriculares diferentes a la Educación Física, de manera que la implementación del modelo de responsabilidad se realice de forma integral en los centros escolares como parte del proyecto educativo, haciéndose extensivo a las distintas situaciones que componen la vida cotidiana del centro, en la línea de recientes investigaciones que ya lo han llevado a cabo con resultados efectivos (Escartí et al., 2018).

Por lo que al perfil del formador se refiere, ha quedado patente que el profesorado participante otorga máxima importancia al conocimiento adquirido sobre la realidad cotidiana de los docentes, del contexto socioeconómico de sus centros y de la población escolar que los integra. Se expone como factor determinante la manera de enseñar, donde una actitud positiva y entusiasta del formador confiere credibilidad a los contenidos, aumenta el grado de motivación y genera un incremento de las expectativas. Otras investigaciones consideran que la experiencia en la aplicación del TPSR debe ser una de las características importantes que debe atesorar el formador (Tarín-Moreno, Pascual \& Escartí, 2013), en este estudio, sin embargo, no ha sido un factor determinante, aunque es obvio que poder aunar la experiencia con el conocimiento del contexto sería lo óptimo para otorgar una formación de mayor calidad.
A modo de conclusión, podemos exponer que un plan de formación en aspectos relacionados con el TPSR, debe tener una carga lectiva de al menos 50 horas que permita desarrollar los niveles de responsabilidad, el modelo de sesión de trabajo, el perfil del docente aplicador, las estrategias de enseñanza de responsabilidad y resolución de conflictos y el entrenamiento de habilidades sociales y de comunicación. Es necesario su adecuación a las características contextuales de los docentes aplicadores, intentado compaginar el proceso de formación con el desarrollo de su labor docente, poniendo especial énfasis en tres elementos: la composición del grupo, favoreciendo la participación; la elección de horarios, ajustándose a las necesidades del profesorado; y las localizaciones, facilitando la asistencia. Además, el plan de formación debe abarcar también el proceso de aplicación de TPSR, pudiéndose extender a otras áreas del currículo y otras etapas. Y por último y no pudiendo ser de otra manera, los testimonios avalan que la labor del formador es fundamental en todo el proceso, quien debe conocer en profundidad el Modelo de Responsabilidad y utilizar un estilo de enseñanza motivador y entusiasta, guiando el proceso de formación a través de elementos prácticos de carácter participativo.

\section{Referencias}

Barbour, R. (2013). Los grupos de discusión en investigación cualitativa. Madrid: Morata.

Beaudion, S. (2012). Using responsibility-based estrategies to empower in-service physical education and health teachers to learn and implement TPSR. Agora para la Educación Física y el Deporte, 14(2), 161-177.

Belando, N., Ferriz-Morell, R., \& Moreno-Murcia, J.A. (2012). Propuesta de un modelo para la mejora personal y social a través de la promoción de la responsabilidad en la actividad físico-deportiva. RICYDE. Revista Internacional De Ciencias Del Deporte, 8(29), 202-222.

Callejo, J. (2001). El grupo de discusión: introducción a una práctica de investigación. Barcelona: Ariel.

Canales, M., \& Peinado, A. (1994). Grupos de discusión. En J.M. Delgado \& J.Gutiérrez (Coords.). Métodos y técnicas cualitativas de investigación en ciencias sociales (287 -316). Madrid: Síntesis.

Cecchini, J., Montero, J., \& Pena, J. (2003). Repercusiones del programa de intervención para desarrollar la responsabilidad personal y social de Hellison sobre los comportamientos de fair-play y autocontrol. Psicothema, 15(4), 631-637.

Coffey, A., \& Atkinson, P. (2005). Encontrar el sentido a los datos cualitativos. Estrategias complementarias de investigación. Alicante: Universidad de Alicante.

Compagnone, N. (1995). Teaching responsibility to rural elementary youth: Going beyond the at-risk boundaries. Journal of Physical Education, Recreation and Dance, 66(6), 58-63.

Debusk, M., \& Hellison, D. (1989). Implementing a physical education self responsibility model for delinquencyprone youth. Journal of Teaching in Physical Education, 8(2), 104.

Escartí, A., Gutiérrez, M., Pascual, C., Marín, D., Martínez, C., \& Chacón, Y. (2006). Enseñando responsabilidad perso- 
nal y social a un grupo de adolescentes de riesgo: Un estudio observacional. Revista de Educación, 341, 373396.

Escartí,A., Gutiérrez, M., Pascual, C., \& Llopis-Goig, R. (2010). Implementation of the personal and social responsibility model to improve self-efficacy during physical education classes for primary school children. International Journal of Psychology and Psychological Therapy, 10(3), 387-402.

Escartí, A., Gutiérrez, M., Pascual, C., \& Wright, P. (2013). Observación de las estrategias que emplean los profesores de educación física para enseñar responsabilidad personal y social. Revista De Psicología Del Deporte, 22(1), 159-166.

Escartí, A., Llopis-Goig, R., \& Wright, P (2018). Assessing the Implementation Fidelity of a School-Based Teaching Personal and Social Responsibility Program in Physical Education and Other Subject Areas. Journal of Teaching in Physical Education, 37, 12-23. doi:org/10.1123/ jtpe.2016-0200

Escartí, A., Pascual, C., \& Gutiérrez, M. (2005). Responsabilidad personal y social a través de la educación física y el deporte. Barcelona: Grao.

Escartí, A., Pascual, C., Gutiérrez, M., Marín, D., Martínez, M., \& Tarín, S. (2012). Applying the teaching personal and social responsibility model (tpsr) in spanish schools context: Lesson learned. Ágora para la Educación Física yel Deporte, 14(2), 178-196.

Fernández-Río, J. (2014). Another step in models-based practice: Hybridizing cooperative learning and teaching for personal and social responsibility. Journal of Physical Education, Recreation and Dance, 85(7), 35. doi:org/10.1080/07303084.2014.937158

Fernández-Río, J., \& Menéndez-Santurio, J. I. (2017). Teachers and Students' Perceptions of a Hybrid Sport Education and Teaching for Personal and Social Responsibility Learning Unit. Journal of Teaching in Physical Education, 36(2), 185-196. doi:org/10.1123/jtpe.2016-0077

Friberg, F., \& Öhlen, J. (2007). Searching for knowledge and understanding while living with impending death-a phenomenological case study. International Journal of Qualitative Studies on Health and Well-being, 2(4), 217226. doi:org/10.1080/17482620701523777

Gómez Mármol, A. (2014). La Responsabilidad Personal y Social, la actividad física y la educación en valores de los escolares de La Región de Murcia. Tesis Doctoral. Universidad de Murcia.

Gordon, B., Jacobs, J., \& Wright, P.M. (2016). Social and emotional learning through a teaching personal and social responsibility based after-school program designed for disengaged middle-school boys. Journal of Teaching in Physical Education, 35, 358-369. doi:10.1123/ jtpe.2016-0106

Gülay, K.A., \& Gürsel, F. (2017). The implementation of personal and social resposibility model in physical education classes: An action research. Egitim Ve Bilim, 42(191). doi:10.15390/EB.2017.7149

Gutiérrez, J. (2008). Dinámica del grupo de discusión. Madrid: CIS.

Heinemann, K. (2003). Introducción a la metodología de la investigación empírica en las ciencias del deporte. Barcelona: Paidotribo.

Hellison, D. (1995). Teaching responsibility through physical activity. Champaign, IL: Human Kinetics.

Hellison, D. (2003). Teaching responsibility through physical activity. ( $2^{\mathrm{a}}$ ed.) Champaign, IL: Human Kinetics.

Hellison, D. (2011). Teaching responsibility through physical activity. ( $3^{\mathrm{a}}$ ed.). Champaign, IL: Human Kinetics.

Ibáñez, J. (2003). Más allá de la sociología. El grupo de discusión: teoría y crítica (5ªd.). Madrid: Siglo XXI.

Jiménez, P.J. (2000). Modelo de intervención para educar en valores a jóvenes en riesgo a través de la actividad física y el deporte. Universidad Politécnica de Madrid.

Jiménez, P. J., \& Durán, J. (2004). Propuesta de un programa para educar en valores a través de la actividad física y el deporte. Apunts: Educación Física y Deportes, 77, 2529.

Kahne, J., Nagaoka, J., Brown, A., O’Brien, J., Quinn, T., \& Thiede, K. (2001). Assessing after-school programs as contexts for youth development. Youth and Society, 32(4), 421-446. doi:org/10.1177/0044118X01032004002

Kallusky, J. (2000). In-school programs. En D. Hellison, N. Cutforth, J. Kallusky, T. Martinek, M. Parker y J. Stiehl (Eds.), Youth development and physical activity: Linking universities and communities, 87-114. Champaign, IL: Human Kinetics.

Kitzinger, J., \& Barbour, R. (1999). Developing Focus Group Research: Politics, Theory and Practice. Londres: Sage.

Krueger, R. (1991). El grupo de discusión. Guía práctica para la investigación aplicada. Madrid: Pirámide.

Lee, O., \& Choi, E. (2015). The Influence of Professional Development on Teachers' Implementation of the Teaching Personal and Social Responsibility Model. Journal of Teaching in Physical Education, 34(4), 603625. doi:10.1123/jtpe.2013-0223

Lee, O., \& Martinek, T. (2009). Navigating two cultures: an investigation of cultures of a responsibility based physical activity program and school. Pedagogy, 80(2), 230-240.

Llopis-Goig, R., Escartí, A., Pascual, C., Gutierrez, M., \& Marín, D. (2011). Strengths, difficulties and improvable aspects in the application of a personal and social responsibility programme in physical education: An evaluation based on the implementers' perceptions. Cultura y Educación, 23(3), 445-461. doi:org/10.1174/113564011797330324

Marín, D. (2011). Adaptación e implementación de un programa de intervención en la escuela a través de la educación física: El programa de responsabilidad personal y social. Tesis Doctoral. Universidad de Valencia.

Martinek, T., \& Hellison, D. (2009). Youth leadership in sport and physical education. Palgrave MacMillan: Nueva York.

Martinek, T., McLaughlin, D., \& Schilling, T. (1999). Project Effort: Teaching responsibility beyond the gym. Journal of Physical Education, Recreation, and Dance, 70(6), 59-65. doi:org/10.1080/07303084.1999.10605954

Martínez, M. (2011). El Desarrollo Positivo a través de la Actividad Física y el Deporte: El Programa de Responsabilidad Personal y Social. Tesis Doctoral no publicada. Universidad de Valencia. 
Menéndez Santurio, J., \& Fernández-Río, J. (2016). Hibridación de los modelos de Educación Deportiva y Responsabilidad Personal y Social: una experiencia a través de un programa de kickboxing educativo. Retos, 30, 150158.

Pardo, R. (2008). La transmisión de valores a jóvenes socialmente desfavorecidos a través de la actividad física y el deporte. Estudio múltiple de casos: Getafe, L'aquila y los ángeles. Tesis Doctoral no publicada. Universidad Politécnica de Madrid.

Pascual, C., Escartí,A., Llopis, R., Gutíerrez, M., Marín, D., \& Wright, P. M. (2011). Implementation fidelity of a program designed to promote personal and social responsibility through physical education: A comparative case study. Research Quarterly for Exercise and Sport, 82(3), 499511.doi:10.1080/02701367.2011.10599783

Patton, M. Q. (2002). Qualitative research and evaluation methods ( $3^{\mathrm{a}}$ ed.). Thousand Oaks, CA: Sage.

Pavão, I., Santos, F., Wright, P.M., \& Gonçalves, F. (2019). Implementing the teaching personal and social responsibility model within preschool education: strengths, challenges and strategies. Curriculum Studies in Health and Physical Education, 10(1) 51-70. doi: 10.1080/25742981.2018.1552499

Pozo, P., Grao-Cruces, A., \& Perez-Ordas, R. (2016). Teaching personal and social responsibility model-based programmes in physical education: A systematic review. European Physical Education Review, 22, 1-20. doi:10.1177/1356336X16664749

Ruiz, M., Rodríguez, P., Martinek, T., Schilling, T., Durán, J., \& Jiménez, P. J. (2006). El Proyecto Esfuerzo: un modelo para el desarrollo de la responsabilidad personal y social a través del deporte. Revista de Educación, 341, 933958.

Saldaña, J. (2009). The Coding Manual for Qualitative Researchers. Thousand Oaks, CA: Sage.

Sánchez-Alcaraz, B. J., Cañadas, Mª ., Valero, A., Gómez, A., \& A., Funes, A. (2019). Results, Difficulties and Improvements in the Model of Personal and Social Responsibility. Apunts. Educación Física y Deportes, 136, 62-82. doi:10.5672/apunts.2014-0983.es.(2019/ 2).136.05

Sánchez-Alcaraz, B. J., Gómez-Mármol, A., Valero, A., De La Cruz, E., \& Esteban, R. (2012). Influencia del Modelo de Responsabilidad Personal y Social en la Calidad de Vida de los Escolares. Cuadernos de Psicología del Deporte, 12 (2), 13-18.

Sánchez-Alcaraz, B. J., Gómez-Mármol, A., Valero, A., \& De La Cruz, E. (2013). Aplicación de un programa para la mejora de la responsabilidad personal y social en las clases de Educación Física. Motricidad. European Journal of Human Movement, 30, 121-129.

Svensson, L., \& Doumas, K (2013). Contextual and Analytic Qualities of Research Methods Exemplified in Research on Teaching. Qualitative Inquiry, 19(6), 441-450. doi:org/ 10.1177/1077800413482097

Schilling, T. (2001). An investigation of commitment among participants in an extended day physical activity program. Research Quarterly for Exercise and Sport, 72(4), 355365. doi:org/10.1080/02701367.2001.10608972
Tarín-Moreno, S., Pascual, C., \& Escartí, A. (2013). La formación en el proceso de implementación del programa de responsabilidad personal y social: un estudio de casos. Revista Fuentes, 14, 125-146.

Vallejo, R., \& Mineira, F. (2009). La triangulación como procedimiento de análisis para investigaciones educativas. Revista Electrónica de Humanidades, Educación y Comunicación Social, 7(4), 117-133.

Vizcarra, M.T. (2004). Análisis de una experiencia de formación permanente en el deporte escolar a través de un programa de habilidades sociales. Tesis Doctoral. Universidad del País Vasco.

Walsh S. (2007) Supporting Youth Development Outcomes: An Evaluation of a Responsibility Model-Based Program. The Physical Educator, 64(1), 48-56.

Walsh S. (2008) Helping youth in underserved communities envision possible futures: an extension of the teaching personal and social responsibility model. Research Quaterly for Exercise and Sport, 79(2), 209-221. doi:org/ 10.1080/02701367.2008.10599484

Walsh S, Ozaeta J., \& Wright M. (2010) Transference of responsibility model goals to the school environment: exploring the impact of a coaching club program. Physical Education and Sport Pedagogy, 15(1), 15-28. doi:10.1080/17408980802401252

Walsh, S., Veri, J., \& Willard, J. (2015). Kinesiology career club: Undergraduate student mentors' perspectives on a physical activity-based teaching personal and social responsibility program. Physical Educator, 72(2), 317323.

Wright, P. M., Li, W., Ding, S., \& Pickering, M. (2010). Integrating a personal and social responsibility program into a Wellness course for urban high school students: assessing implementation and educational outcomes. Sport, Education and Society, 15(3), 277-298. doi:10.1080/ 13573322.2010.493309

Wright, P. M., White, K., \& Gaebler-Spira, D. (2004). Exploring the relevance of the personal and social responsibility model in adapted physical activity: a collective case study. Journal of teaching in physical educations, 23, 71-87. doi:10.1123/jtpe.23.1.71

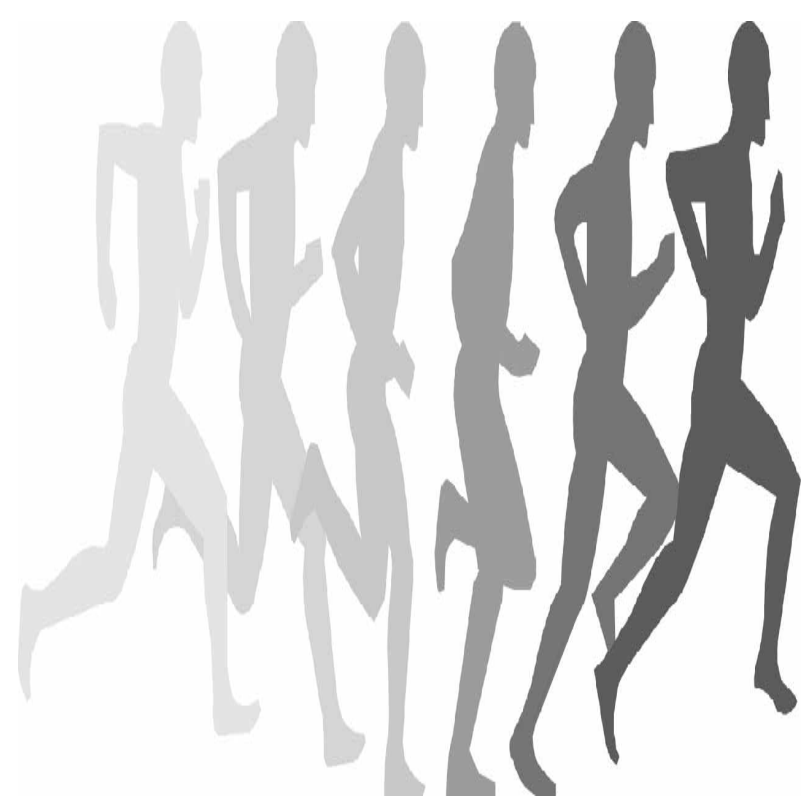

